\title{
Nevoid Acanthosis Nigricans Located on the Scalp
}

\author{
Jorge Román-Sainz ${ }^{1}$, Nicolás Silvestre-Torner ${ }^{1}$, Sergio Samer Tabbara-Carrascosa ${ }^{1}$, Adrián \\ Imbernón-Moya ${ }^{1}$
}

1 Dermatology Unit, Hospital Universitario Severo Ochoa, Leganés, Madrid, Spain Citation: Román-Sainz J, Silvestre-Torner N, Tabbara-Carrascosa SS, Imbernón-Moya A. Nevoid acanthosis nigricans located on the scalp.
Dermatol Pract Concept. 2022;12(1):e2022009. DOI:https://doi.org/10.5826/dpc.1201a09

Accepted: May 6, 2021; Published: January 2022

Copyright: @2022 Román-Sainz et al. This is an open-access article distributed under the terms of the Creative Commons AttributionNonCommercial License (BY-NC-4.0), https://creativecommons.org/licenses/by-nc/4.0/, which permits unrestricted noncommercial use, distribution, and reproduction in any medium, provided the original authors and source are credited.

Funding: None.

Competing interests: None.

Authorship: All authors have contributed significantly to this publication.

Corresponding author: Jorge Román-Sainz, Dermatology Unit, Hospital Universitario Severo Ochoa, Leganés, Madrid, Spain. E-mail: jorgesheldon@gmail.com

\section{Case Presentation}

A 14-year-old male presented with an alopecic plaque in his occipital area. Skin exam revealed a well-defined, hyperpigmented plaque with a velvety texture, resembling acanthosis nigricans (AN) (Figure 1A). Trichoscopy showed broken hair shafts and yellow dots, as well as a few exclamation mark hairs. Histology manifested mild acanthosis, hyperkeratosis, and papillomatosis, with no visible nests of melanocytes (Figure 1B). Due to these findings, the patient was diagnosed with nevoid AN. This skin disorder, also known as RAVEN (round and velvety epidermal nevus) is a rare entity, which appears at or before puberty $[1,2]$.

The skin disorder RAVEN has no predilection for intertriginous or flexural areas. Most reported cases are unilateral and located on the chest, abdomen and umbilical, or retroauricular area. We found no other reports of RAVEN involving the scalp in the literature. As it is a benign condition, RAVEN requires no treatment or follow-up, and has not been found to be associated with any underlying disorder [1,2].
The closest differential diagnosis is with epidermal nevus. Although they are clinically very similar, RAVEN is usually velvetier, and histology plays an essential role as it shows compact hyperkeratosis, papillomatosis and limited acanthosis as compared to epidermal nevi. Other differential diagnosis include confluent and reticulate papillomatosis, ichthyosis hystrix, and the hyperkeratotic type of seborrheic keratosis. In our case, the velvety texture of the lesion, as well as the histology, which showed mild acanthosis, excluded epidermal nevus.

\section{Teaching Point}

RAVEN can resemble epidermal nevi or AN. Dermatologists should be aware of this entity and should consider it in the differential diagnosis of pigmented skin lesions.

Informed consent: Informed consent for publication of clinical details and clinical images was obtained from the patient. 


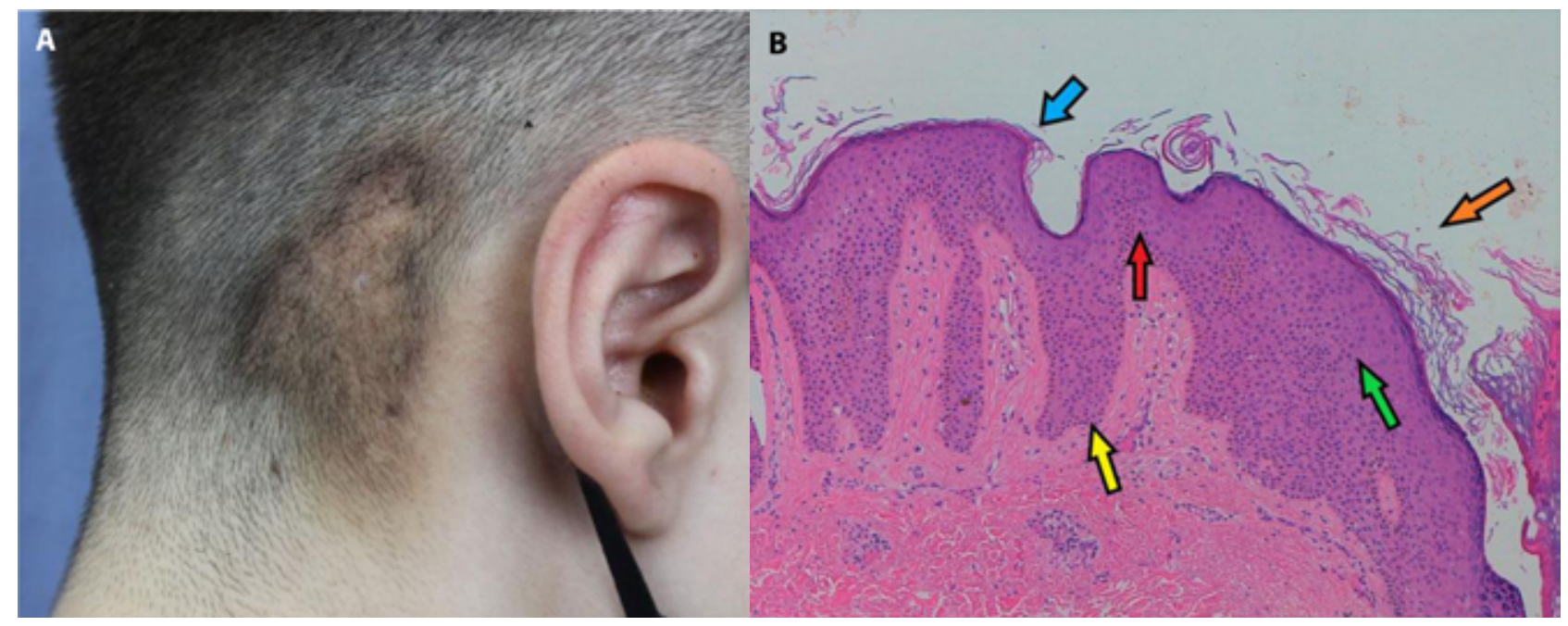

Figure 1. (A) Well-defined, hyperpigmented plaque located in the occipital area of the patient, associated with loss of hair density. (B) Histology showing elongated papilla (yellow arrow), acanthosis (green arrow), hypergranulosis (blue arrow) and hyperkeratosis (orange arrow). Melanosis (red arrow) is also visible in some areas.

\section{References}

1. Petit A, Lemarchand-Venenciea F, Pinquier L, Lebbea C, Bourrat E. Nevoid acanthosis nigricans or RAVEN (rounded and velvety epidermal nevus): three cases. Ann Dermatol Venereol. 2012; 139(3):183-188. DOI: 10.1016/j.annder.2011.10.411. PMID: 22401682.

2. Larsabal M, Cogrel O, Caumont C, Jegou MH, Taïeb A, Morice-Picard F. Mosaic mutations in FGFR3 and FGFR2 are associated with naevoid acanthosis nigricans or RAVEN (round and velvety epidermal naevus). Br J Dermatol. 2019;180(1): 201-202. DOI: 10.1111/bjd.17095. PMID: 30117157. 